\title{
Space-Charge Compensation Options for the LHC Injector Complex *
}

\author{
M. Aiba, C. Carli, M. Chanel, U. Dorda, R. Garoby, J.-P. Koutchouk, M. Martini, E. Metral, \\ Y. Papaphilippou, W. Scandale, F. Zimmermann, CERN; V. Shiltsev, FNAL; G. Franchetti, GSI
}

\begin{abstract}
Space-charge effects have been identified as the most serious intensity limitation in the CERN PS and PS Booster, on the way towards ultimate LHC performance and beyond. We here explore the application of several previously proposed space-charge compensation methods to the two LHC pre-injector rings, and the challenges which need to be overcome. The methods considered include the reduction of tune shift and resonance strengths via octupoles, pole-face windings, electron lenses, or neutralisation.
\end{abstract}

\section{INTRODUCTION}

The bunch intensity which can be injected into the Large Hadron Collider (LHC) will be limited by space-charge (s.c.) effects in its injector complex. The two LHC injector rings strongly afflicted by s.c. are the Proton Synchrotron (PS) and the PS Booster. As a result of the booster's low injection energy of $50 \mathrm{MeV}$, the vertical incoherent tune spread due to s.c. will reach a value of 0.5 for the beam needed to deliver the so-called "ultimate" intensity of $1.7 \times$ $10^{11}$ protons per bunch to the LHC [1]. The PS uses a "double batch" injection, during which the $1.4-\mathrm{GeV}$ beam spends $1.2 \mathrm{~s}$ (about $5 \times 10^{5}$ turns) on the injection plateau, which reduces the maximum tune spread acceptable in this machine to approximately 0.25 [1].

In both the PS Booster and PS, s.-c. effects manifest themselves in the form of beam loss, core emittance growth, and halo formation. The brute-force approach to overcoming s.-c. bottlenecks is the upgrade of the entire injector complex, as is planned. A new injector linac will first raise the injection energy into the booster to $160 \mathrm{MeV}$ and, in its final incarnation, make the booster fully obsolete [2]. The PS itself will be replaced by a larger-circumference ring, "PS2", with a higher injection energy of $5 \mathrm{GeV}$ [2]. Until this upgrade is completed, an intermediate solution could be the compensation of s.-c. effects in the existing rings. Various possibilities are surveyed in this paper. Estimates are made based on Table 1, which lists approximate beam parameters of the PS and the PS Booster at the ultimate LHC intensity, and also shows that the injectors are not far from delivering the ultmate LHC beam, hinting that a partial s.-c. compensation may be sufficient.

Assuming a Gaussian transverse distribution, the maximum value of the direct s.-c. tune shift is

$$
-\Delta Q_{x, y}^{S C}=\frac{N_{t} r_{p} B_{f}}{4 \pi \beta_{p}^{2} \gamma_{p}^{3}} \int \frac{\beta_{x, y} d s}{\sigma_{x, y}\left(\sigma_{x}+\sigma_{y}\right)} \approx \frac{N_{b} r_{p} B_{f}}{4 \pi \epsilon_{N} \beta_{p} \gamma_{p}^{2}},
$$

where $\beta_{p}=v_{p} / c$ and $\gamma_{p}$ are the relativistic parameters, ${ }^{(1)}$ is the classical proton radius, $B_{f}$ the bunching factor (ratio

\footnotetext{
${ }^{*}$ We acknowledge the support of the European Community-Research Infrastructure Initiative under the FP6 "Structuring the European Research Area" programme (CARE, contract number RII3-CT-2003-506395).
}

Table 1: Parameters of the CERN PS and the PS Booster (PSB) proton beams corresponding to the "ultimate" LHC.

\begin{tabular}{lccc}
\hline variable & symbol & PSB & PS \\
\hline kin. energy & $E_{\text {kin }}$ & $50 \mathrm{MeV}$ & $1.4 \mathrm{GeV}$ \\
circumference & $C$ & $157 \mathrm{~m}$ & $628 \mathrm{~m}$ \\
protons/bunch & $N_{b}$ & $2.5 \times 10^{12}$ & $2.5 \times 10^{12}$ \\
protons/beam & $N_{\mathrm{t}}$ & $2.5 \times 10^{12}$ & $1.5 \times 10^{13}$ \\
tr. n. emittance & $\beta \gamma \epsilon$ & $2.5 \mu \mathrm{m}$ & $3 \mu \mathrm{m}$ \\
full bunch length & $l_{b} / c$ & $750 \mathrm{~ns}$ & $180 \mathrm{~ns}$ \\
harmonic number & $h$ & $1(\& 2)$ & 7 \\
av. beta function & $\beta_{x, y}$ & $5 \mathrm{~m}$ & $15 \mathrm{~m}$ \\
superperiodicity & $P$ & 16 & 10 \\
betatron tunes & $Q_{x, y}$ & $4.29,5.45$ & $6.12,6.24$ \\
revolution period & $T_{0}$ & $1.7 \mu \mathrm{s}$ & $2.3 \mu \mathrm{s}$ \\
bunching factor & $B_{f}$ & 2.2 & 3.4 \\
s.c. tune shift & $\Delta Q^{S C}$ & 0.76 & 0.35 \\
\hline
\end{tabular}

of peak current to average current), $\sigma_{x, y}$ the rms beam size, and $\beta_{x, y}$ the beta function. The approximation (1) was used to estimate the s.-c. tune shifts in Table 1.

\section{COMPENSATION TECHNIQUES}

Octupole fields could cancel the next-to-leading term in the s.-c. force. For a round beam, the 4th order term of the direct s.-c. potential varies as $\left(x^{4}+2 x^{2} y^{2}+y^{4}\right)$, while the potential of an octupole is proportional to $\left(x^{4}-\right.$ $\left.6 x^{2} y^{2}+y^{4}\right)$. Therefore, at least two families of octupoles are needed to reduce the s.-c. tune spread, which are placed at locations with either peak and intermediate values of the beta function, respectively. The beta functions should sufficiently vary over the length of an optical cell, e.g., by a factor 2 or more.

Defining a normalized octupole strength as $K_{\text {oct }} \equiv$ $\left(\partial^{3} B_{y}\right) /\left.\left(\partial x^{3}\right)\right|_{0} l_{\text {oct }} /(B \rho)$, where $(B \rho)$ denotes the magnetic rigidity, and $l_{\text {oct }}$ the length of the octupole, the minimum octupole strength required to cancel the linear tune shift with action ( $\propto$ squared amplitude) is of order $K_{\text {oct }} \approx$ $6 \pi \Delta Q^{S C} /\left(\beta_{x, y} \sigma_{x, y}^{2}\right)$. Inserting the parameters of Table 1 we find values of $7.6 \times 10^{4} \mathrm{~m}^{-3}$ for the booster and $2.3 \times 10^{4} \mathrm{~m}^{-3}$ for the PS.

Pole-face windings allow precise adjustments of the tune shift with transverse position up to a high order. At the ISR, 24 pole-face windings modifying the local magnetic field were used to correct the horizontal and vertical tune shift plus the next 4 orders in their Taylor expansions with respect to the horizontal position [3]. The correction combined an analysis of the produced field pattern in terms of multipole components and a study of the influence of the excitation of each individual circuit [4]. The s.-c. detuning was corrected online, based on beam-transfer function measurements and longitudinal Schottky signals [5], which both provided information on the beam distribution 
as a function of the horizontal position. The incoherent direct and indirect s.c. tune shifts for the ISR were modelled by theoretical expressions [3], which translated into curves for different horizontal beam offsets that guided the s.-c. correction applied for every $3 \mathrm{~A}$ of beam stacking. This technique increased the ISR beam current 15 times.

Electron lenses, in which a negatively charged electron beam collides with the proton beam inside a strong solenoid field, could also compensate the s.-c. tune shift $[6,7]$. The devices needed would be similar to the two Tevatron electron lenses actually used for beam-beam compensation [8]. Assuming $N_{l}$ electron lenses, each of length $L$, distributed around the ring, and an electron beam copropagating with the proton beam, the electron current needed per lens is [6] $J_{e}=B_{f} \kappa e c N_{\mathrm{t}} /\left(L N_{l}\right) \beta_{e} /\left(\gamma_{p}^{2}(1-\right.$ $\left.\beta_{p} \beta_{e}\right)$ ), where $\kappa$ denotes the degree of compensation. Assuming an electron kinetic energy of $10 \mathrm{keV}$, and considering four or five 2-m long lenses per ring, at the "optimum" compensation level $\kappa \approx 30 \%$ of [6], each lens should provide an electron current of about $1.2 \mathrm{~A}$ or $2.3 \mathrm{~A}$ for the booster and the PS, respectively. This is close to the maximum current delivered by the operating Tevatron electron lenses. Figure 1 illustrates that four electron lenses in the PS Booster can effectively reduce the s.-c. tune spread, at moderate beam intensity $(\sim 1 / 2$ the nominal).

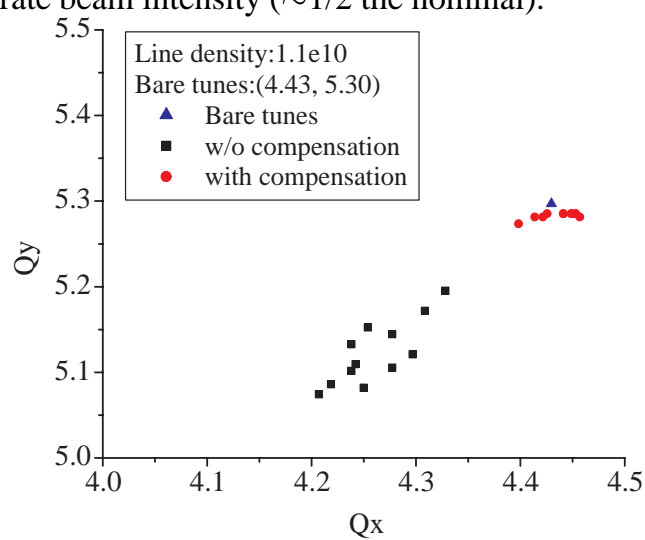

Figure 1: Tune footprint of the PS Booster computed for 12 test particles launched on a 2-D grid with initial start amplitudes ranging from $1 \sigma$ to $2 \sigma$, without and with s.c. compensation by 4 electron lenses.

In case of passive neutralisation the s.-c. force of a proton beam is partly compensated by ionization electrons, electron cloud, or negative ions (the latter are less likely since they would easily change their charge state when hit by the beam) which are approximately at rest longitudinally, but move transversely during the beam passage. This type compensation was first proposed by G.I. Budker [9], a theoretical analysis of coherent stability limits in such system was performed by B.V. Chirikov [10], and an experimental demonstration of overcoming (coherent) s.-c. limitations by increasing the residual gas density was finally realized at the INP PSR $[11,12]$.

Since the ionization electrons do not move longitudinally with the beam particles, the electron line density needed for compensation is only a small fraction $\eta$ of the beam line density: The compensation condition reads $\eta \approx 1 / \gamma_{p}^{2}$ $[9,3]$. At the ISR, various measurements indicated that, at $26 \mathrm{GeV}$, the natural beam neutralisation from residual gas ionization cancelled the direct s.-c. force, i.e., $\eta \approx 10^{-3}$. As a result, the direct s.c. effect could be ignored. However, due to the much lower beam energy, for the PSB we would need $\eta \approx 0.9$ and for the PS injection $\eta \approx 0.16$. The corresponding large electron densities could lead to electronproton instabilities.

Optimum compensation requires that the transverse electron and beam distributions are matched. For bunched beam, this could be achieved by confining the electrons with strong solenoid fields to "columns," and using electrostatic electrodes to fine tune the charge density [13].

\section{CHALLENGES}

Bunch head and tail are likely to experience an overcompensation of their s.-c. tune shift by the octupoles, resulting in an overall tune shift of opposite sign. This overcompensation for parts of the beam and the associated resonance crossing (see also [14]) may explain why past attempts to decrease the s.-c. tune spread at the PS Booster were not successful [15]. For a perfectly flat longitudinal bunch profile or for a coasting beam, as in the ISR, the unwanted effect would disappear.

For the same reason, the electron current of a lens compensator must be rapidly modulated to match the longitudinal profile of each (non-flat) bunch [6]. The peak electron current of the lens compensation could be reduced by a factor $\left(1+\beta_{e} \beta_{p}\right) /\left(1-\beta_{e} \beta_{p}\right)$ [or $10-40 \%$ ] if the electron beam were sent in the direction opposite to the proton beam. However, this would put stronger demands on the modulation speed. Figure 2 illustrates the emittance growth caused by a single electron lens without current modulation for a PS-like ring. At a moderate s.-c. tune shift of 0.1 , the emittance stays approximately constant. For a tune shift of $\Delta Q^{S C} \approx 0.13$, particles at a longitudinal distance $\pm 3 \sigma_{z}$ from the bunch center cross the half integer resonance. For further increasing s.-c. tune shift the point of resonance crossing moves closer and closer towards the bunch center, resulting in ever larger emittance growth. This effect can be mitigated by increasing the number of lenses. The minimum number of compensators $N_{c}$ needed follows from the stability criterion

$$
\left|\cos \phi_{0}+\sin \phi_{0}\left(\frac{Q}{Q_{0}} \frac{\left(\cos \phi_{0}-\cos \phi\right)}{\sin \phi}\right)\right|<1,
$$

where $\phi \equiv 2 \pi Q / N_{c}, \phi_{0} \equiv 2 \pi Q_{0} / N_{c}, Q \equiv Q_{0}+\Delta Q^{S C}$. Another issue is the general "stability" of the electron-lens compensation. Resonance crossing, due to either non-ideal current modulation or the discrete compensator locations, can lead to transverse amplitude growth at the longitudinal edges of the bunch, which in turn, a quarter synchrotron period later, will alter the s.-c. tune shift at the bunch center. This enhances the residual tune spread, rendering even more particles unstable. The intensity and size of the electron lens may therefore require continuous adjustment.

In case of passive neutralisation, the negatively charged particles are accelerated transversely in the field of the 

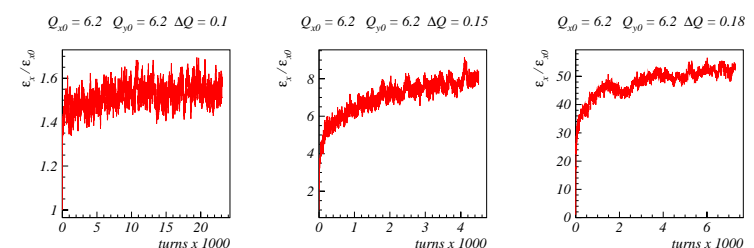

Figure 2: Horizontal emittance growth in a PS-like ring for a single constant electron lens adjusted to compensate a peak s.-c. tune shift $\Delta Q^{S C}$ of 0.1 (left), 0.15 (center) and 0.18 (right). Particles in the head and tail are overcompensated, and their transverse motion becomes unstable for $\Delta Q^{S C}>0.13$.

beam, and, for a bunched beam, their transverse distribution can be matched to that of the beam only "on average", while over- and undercompensation occur at the bunch tail and head, respectively. A likely arising spiky electron distribution at the bunch tail may also introduce significant nonlinear field components. Again the s.-c. compensation appears easier for unbunched beams, where a matched equilibrium distribution of trapped electrons is automaticaly established. A possible solution for bunched beams are the magnetized electron columns [13].

Either coherent modes or incoherent processes could lead to the observed s.-c. limits; see e.g. [6]. In the CERN machines, no evidence of coherent instabilities is seen during normal operation. Simulations of s.-c. dynamics in the PS Booster are presently ongoing with two independent codes (ACCSIM and ORBIT) [16]. For an elliptical beam distribution, one of the two (ORBIT) shows occasional short bursts of coherent motion which contribute to the otherwise incoherent emittance growth; these bursts do not occur for a Gaussian beam (Fig. 3).
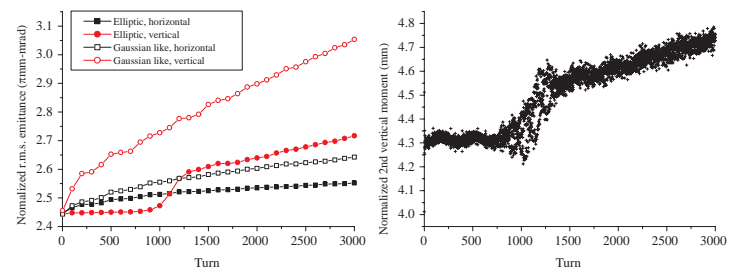

Figure 3: Emittance growth for a Gaussian and an elliptical distribution in the PS Booster (left), and the beam size for the latter case (right), as a function of turn number, simulated by ORBIT. A period of steeper growth coincides with transverse coherent beam-size oscillations.

Based on the coherent tune shifts of the dipole mode and of two envelope modes computed for a uniform beam distribution, the authors in [6] concluded that the best compensation achievable with an electron lens is about $30 \%$. Due to the sharpe edge of the beam density, this model may exaggerate coherent instabilities. The coherent tune shifts for more realistic distributions in the presence of an electron lens could be investigated either by solving the pertinent Vlasov equation or by including an electron lens in the ORBIT simulation code, which is planned.

Resonances can be excited more strongly by the discrete locations of nonlinear compensation elements placed around the ring. In [17] higher-order resonance strengths as a function of transverse amplitude for various radial beam distributions of an electron cooler were calculated, which would also apply to an electron lens. In addition, due to symmetry, strong and wide linear "structure" resonances occur for $2 Q_{x, y}=m P$, where $m$ denotes an arbitrary integer and $P$ the superiodicity of the ring. The latter should ideally be preserved by the compensation scheme, in order not to dramatically increase the number of harmful resonances close to the operating point. For instance, using 4 symmetrically placed lenses in the PS Booster reduces the superperiodicity $P$ from 16 to 4 , and $2 Q_{x}=8$ then becomes a structure resonance, leading to a failure of the electron-lens compensation at high beam intensity (Fig. 4).
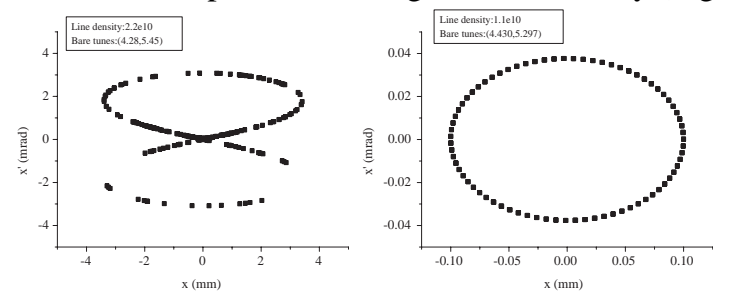

Figure 4: Particle trajectory in horizontal phase space at a peak proton line density of $2.2 \times 10^{10} \mathrm{~m}^{-1}$ [or $N_{b} \approx 2 \times$ $10^{12}$ ] (left) and $1.1 \times 10^{10} \mathrm{~m}^{-1}$ (right) for the PS Booster with 4 electron lenses. At high intensity, the horizontal phase space is destabilized by the newly excited structure resonance $2 Q_{x}=8$.

\section{CONCLUSIONS}

Various techniques could alleviate s.-c. effects in the LHC injector complex and help to increase the beam brightness. They face a number of obstacles, such as overcompensation at the head and tail of the bunch, superperiodicity breaking, resonance excitation, and coherent instabilities. The use of electron lenses for s.-c. compensation deserves further studies. It is encouraging that nonlinear s.c. correction and beam neutralisation were successfully applied with coasting beam at several machines.

\section{REFERENCES}

[1] G. Arduini, Proc. LUMI'06 Valencia.

[2] R. Garoby, Proc. LUMI'06, Valencia.

[3] P.J. Bryant et al, CERN ISR-MA/75-54.

[4] J.P. Gourber, K.N. Henrichsen, CERN ISR-MA/71-38.

[5] J. Borer et al, CERN ISR-RF/80-03.

[6] A.V. Burov, G.W. Foster, V.D. Shiltsev, PAC 2001, Chicago.

[7] W. Scandale, F. Zimmermann, "Injector Upgrade," Proc. LUMI'06 Valencia.

[8] V. Shiltsev et al, PAC'05 Knoxville.

[9] G.I. Budker, Atomic Energy 1, 5 (1956)

[10] B.V. Chirikov, Atomic Energy 19, 3 (1965)

[11] G. Dimov, V. Chupriyanov, Part. Acc. 14, 155 (1984)

[12] V. Dudnikov, ICFA-HB2002, AIP CP 642.

[13] V. Shiltsev, FNAL beams-doc-2810.

[14] G. Franchetti, TUZAAB02, this conference.

[15] M. Chanel, unpublished.

[16] F. Gerikg et al, TUPAN093, this conference.

[17] V. Ziemann, NIM A 556 (2006). 